[13] D. McRuer, I. Ashkenas, and D. Graham, Aircraft Dynamics and Automatic Control. Princeton, NJ: Princeton Univ. Press, 1973.

[14] I. Yaesh, U. Shaked, and T. Yossef, "Simplified adaptive control of F16 aircraft pitch and angle-of-attack loops," in Proc. 44st Israel Annu. Conf. Aerosp. Sci., Feb. 25-26, 2004, [CD ROM].

[15] N. Levanon, Radar Principles. New York: Wiley, 1988.

[16] P. Gahinet, A. Nemirovsky, A. J. Laub, and M. Chilali, LMI Control Toolbox for Use with MATLAB. Natick, MA: The Mathworks, Inc., 1995.

\section{Exponential Stability of a Class of Boundary Control Systems}

Javier Andres Villegas, Hans Zwart, Yann Le Gorrec, and Bernhard Maschke

\begin{abstract}
We study a class of partial differential equations (with variable coefficients) on a one dimensional spatial domain with control and observation at the boundary. For this class of systems we provide simple tools to check exponential stability. This class is general enough to include models of flexible structures, traveling waves, heat exchangers, and bioreactors among others. The result is based on the use of a generating function (the energy for physical systems) and an inequality condition at the boundary. Furthermore, based on the port Hamiltonian approach, we give a constructive method to reduce this inequality to a simple matrix inequality.
\end{abstract}

Index Terms-Boundary control systems (BCS), partial differential equations (PDEs).

\section{INTRODUCTION}

The abstract class of systems which models partial differential equations (PDEs) with control at the boundary of its spatial domain is know as boundary control systems (BCS). The analysis and modeling of this type of systems dates back to the sixties with the leading work of Fattorini [1], and it has become a well established and rich field. Significant research has been carried out in the works of Balakrishnan ([2], [3]), Lions [4], Lasiecka and Triggiani [5], Curtain and Pritchard ([6], [7]), just to name a few. Stability, controllability and observability concepts are subtle in this area and investigating these for a single PDE example leads, in many cases, to a sophisticated mathematical problem. This technical note aims at facilitating the verification of the exponential stability of a class of boundary control systems that arises from the port-Hamiltonian approach to distributed parameter systems [8]. This verification might be translated into an easily checkable matrix condition.

Manuscript received February 27, 2008; revised July 16, 2008, July 17, 2008, and September 23, 2008. Current version published January 14, 2009. This work was supported by NWO, Project ERACIS, 613.000 .223 , by the European sponsored project GeoPlex under Reference Code IST-2001-34166, and by French NRA sponsored project RECIPROC under Reference Code ANR-06JCJC-0011. Recommended by Associate Editor K. A. Morris.

J. A. Villegas is with the Department of Engineering Science, University of Oxford, Oxford OX1 3PJ, U.K. (e-mail: javier.villegas@eng.ox.ac.uk).

H. Zwart is with the Department of Applied Mathematics, Faculty of Electrical Engineering, Mathematics and Computer Science, University of Twente, Enschede 7500 AE, The Netherlands (e-mail: h.j.zwart@math.utwente.nl).

Y. Le Gorrec and B. Maschke are with LAGEP-UMR CNRS, Université de Lyon, Villeurbanne cedex 69622, France, (e-mail: legorrec@lagep.univlyon1.fr; maschke@lagep.univ-lyon1.fr).

Digital Object Identifier 10.1109/TAC.2008.2007176
One of the main concerns in the analysis of PDEs is stability. It is impossible to discuss here all the results which were obtained on this stability problem, and we refer the interested reader to the books [2], [5], [4], [7], [9]-[13] and the references therein. Two of the most common approaches to check the exponential stability property are the harmonic analysis (see [11] and the references therein) and the multiplier method [12]. The multiplier method relies on a proper choice of one (or more) function with certain properties (multiplier), which is central to the solution of the stability problem for a specific PDE model. In this technical note we combine ideas from the multiplier method and recent results on boundary control systems [14] in order to prove exponential stability. The key point is that the multiplier constructed for the wave equation by Cox and Zuazua [15] works after some modifications for our class of BCS. This class is obtained by using the underlying geometric structure together with the energy function of the system. All this is based on the port-Hamiltonian approach to distributed parameter systems, [8], which allows to define general classes of systems. In fact, the class of systems we study is general enough to include models of flexible structures, traveling waves, heat exchangers, bioreactors, and, in general, (lossless and dissipative) hyperbolic systems in one-dimensional spatial domain. For a more complete perspective on significant PDE classes and boundary control problems see, for instance, [9] and [10].

Here $M_{n}(\mathcal{H})$ denotes the space of square $n \times n$ matrices whose entries lie in the vector space $\mathcal{H}$. By $\langle\cdot, \cdot\rangle_{\mathbb{R}}$ we denote the inner product on $\mathbb{R}$ or $\mathbb{R}^{n}$, and $\langle\cdot, \cdot\rangle_{L_{2}}$ (or simply $\langle\cdot, \cdot\rangle$ ) denotes the standard inner product on $L_{2}\left(a, b ; \mathbb{R}^{n}\right)$. The Sobolev space of order $k$ is denoted by $H^{k}\left(a, b ; \mathbb{R}^{n}\right)$. We say that a symmetric matrix $M$ is positive definite (in short $M>0)$ if all its eigenvalues are positive, and positive semidefinite (in short $M \geq 0$ ) if its eigenvalues are nonnegative. A self-adjoint operator $L$ is coercive (or strictly positive) on an inner product space $X$ if there exists an $\varepsilon>0$ such that $L \geq \varepsilon I$.

The technical note is organized as follows. In the next section we describe briefly some of the main ideas that will be used to prove the main results. In Section III we present the main results on exponential stability. Section IV presents some examples. Finally, in Section V we give some conclusions.

\section{SOME BACKGROUND}

Most of the results described in this section can be found in [14] and [16]. In this technical note we study systems described by the following PDE:

$$
\frac{\partial x}{\partial t}(t, z)=P_{1} \frac{\partial}{\partial z}(\mathcal{L}(z) x(t, z))+\left(P_{0}-G_{0}\right) \mathcal{L}(z) x(t, z)
$$

$z \in[a, b]$, satisfying the following assumption.

Assumption II.1: $P_{1} \in M_{n}(\mathbb{R})$ is a nonsingular symmetric matrix, $P_{0}=-P_{0}^{T} \in M_{n}(\mathbb{R}), G_{0} \in M_{n}(\mathbb{R})$ is positive semidefinite $\left(G_{0} \geq\right.$ 0 ), and $x$ takes values in $\mathbb{R}^{n}$. Furthermore, $\mathcal{L}(\cdot) \in M_{n}\left(L_{2}(a, b)\right)$ is a bounded and continuously differentiable matrix-valued function satisfying for all $z \in[a, b], \mathcal{L}(z)=\mathcal{L}(z)^{T}$ and $\mathcal{L}(z) \geq m I$, with $m$ independent of $z$.

Note that $\mathcal{L}(z)$ is only assumed to depend on the variable $z$ and not on time. For simplicity, $\mathcal{L}(z) x(t, z)$ will be denoted by $(\mathcal{L} x)(t, z)$, even though $\mathcal{L}$ depends only on $z$. The state space is defined as

$$
\begin{aligned}
X & =L_{2}\left(a, b ; \mathbb{R}^{n}\right) \text { with inner product }\left\langle x_{1}, x_{2}\right\rangle_{\mathcal{L}} \\
& =\left\langle x_{1}, \mathcal{L} x_{2}\right\rangle \text { and norm }\left\|x_{1}\right\|_{\mathcal{L}}^{2}=\left\langle x_{1}, x_{1}\right\rangle_{\mathcal{L}}
\end{aligned}
$$

where $\langle\cdot, \cdot\rangle$ is the natural $L_{2}$-inner product. Hence $X$ is a Hilbert space. By our assumptions on $\mathcal{L}$, it is easy to see that the natural norm on $\mathrm{X}$ and the $L_{2}$-norm are equivalent. The reason for selecting this state 
space is that $\|\cdot\|_{\mathcal{L}}^{2}$ is usually proportional to the energy function of the system. That is why $X$ is sometimes known as the energy state space and the variable $\mathcal{L} x$ is called co-energy variable.

In [14] the authors study a class of BCS that includes the PDE (1) with $G_{0}=0$ (in fact, they also consider higher order differential operators) which corresponds to lossless hyperbolic systems. They provide a complete parameterization of the boundary conditions generating a unitary or contraction semigroup and the associated linear BCS ${ }^{1}$. An important feature of this construction is that it is based on matrix inequalities which are easily checked knowing the matrix differential operator. In [16] these ideas have been extended to a larger class of operators including parabolic and (dissipative) hyperbolic systems all described in one class of systems. Based on that we introduce the boundary portvariables which are related to (1). These port-variables help us to define BCS, in particular, the input and output of the system.

Definition II.2: Let $\mathcal{L} x \in H^{1}\left(a, b ; \mathbb{R}^{n}\right)$. Then, the boundary port variables associated with the system (1) are the vectors $e_{\partial, \mathcal{L} x}, f_{\partial, \mathcal{L} x} \in$ $\mathbb{R}^{n}$, defined by

$$
\left(\begin{array}{l}
f_{\partial, \mathcal{L} x} \\
e_{\partial, \mathcal{L} x}
\end{array}\right)=\frac{1}{\sqrt{2}}\left[\begin{array}{cc}
P_{1} & -P_{1} \\
I & I
\end{array}\right]\left[\begin{array}{l}
(\mathcal{L} x)(b) \\
(\mathcal{L} x)(a)
\end{array}\right] .
$$

Note that the port-variables are nothing else than a linear combination of the boundary variables. We also define the matrix $\Sigma \in M_{2 n}(\mathbb{R})$ as follows

$$
\Sigma=\left[\begin{array}{ll}
0 & I \\
I & 0
\end{array}\right]
$$

Let $W$ and $\tilde{W}$ be two $n \times 2 n$ matrices. It is easy to see that the matrix

$$
\left[\begin{array}{l}
W \\
\tilde{W}
\end{array}\right] \Sigma\left[\begin{array}{l}
W \\
\tilde{W}
\end{array}\right]^{T}=\left[\begin{array}{ll}
W \Sigma W^{T} & W \Sigma \tilde{W}^{T} \\
\tilde{W} \Sigma W^{T} & \tilde{W} \Sigma \tilde{W}^{T}
\end{array}\right]
$$

is invertible if and only if $\left[\begin{array}{l}W \\ \widetilde{W}\end{array}\right]$ is invertible. In [14] (for the case $G_{0}=0$ ) and [16] the authors prove the following theorem.

Theorem II.3: Let $W$ be a $n \times 2 n$ real matrix. If $W$ has full rank and satisfies $W \Sigma W^{T} \geq 0$, where $\Sigma$ is defined in (4), then the system (satisfying Assumption II.1)

$$
\frac{\partial x}{\partial t}(t, z)=P_{1} \frac{\partial}{\partial z}(\mathcal{L} x)(t, z)+\left(P_{0}-G_{0}\right) \mathcal{L} x(t, z)
$$

with input

$$
u(t)=W\left[\begin{array}{l}
f_{\partial, \mathcal{L}_{x}}(t) \\
e_{\partial, \mathcal{L}_{x}}(t)
\end{array}\right]
$$

is a boundary control system on $X$. Furthermore, the operator $\mathcal{A} x=$ $P_{1}(\partial / \partial z)(\mathcal{L} x)+\left(P_{0}-G_{0}\right) \mathcal{L} x$ with domain

$$
D(\mathcal{A})=\left\{\mathcal{L} x \in H^{1}\left(a, b ; \mathbb{R}^{n}\right) \mid\left[\begin{array}{l}
f_{\partial, \mathcal{L} x} \\
e_{\partial, \mathcal{L} x}
\end{array}\right] \in \operatorname{ker} W\right\}
$$

generates a contraction semigroup on $X$.

Let $\widetilde{W}$ be a full rank matrix of size $n \times 2 n$ with $\left[\begin{array}{l}W \\ \widetilde{W}\end{array}\right]$ invertible and let $P_{W, \tilde{W}}$ be given by

$$
P_{W, \tilde{W}}=\left[\begin{array}{ll}
W \Sigma W^{T} & W \Sigma \widetilde{W}^{T} \\
\widetilde{W} \Sigma W^{T} & \widetilde{W} \Sigma \widetilde{W}^{T}
\end{array}\right]^{-1} .
$$

Define the linear mapping $\mathcal{C}: \mathcal{L}^{-1} H^{1}\left(a, b ; \mathbb{R}^{n}\right) \rightarrow \mathbb{R}^{n}$ as,

$$
\mathcal{C} x(t):=\widetilde{W}\left[\begin{array}{l}
f_{\partial, \mathcal{L}_{x}}(t) \\
e_{\partial, \mathcal{L}_{x}}(t)
\end{array}\right]
$$

${ }^{1}$ For the definition of boundary control system (BCS) refer to [9]. and the output as

$$
y(t)=\mathcal{C} x(t) .
$$

Then for $u \in C^{2}\left(0, \infty ; \mathbb{R}^{k}\right), \mathcal{L} x(0) \in H^{1}\left(a, b, \mathbb{R}^{n}\right)$, and $u(0)=$ $W\left[\begin{array}{l}f_{\partial, \mathcal{L} x}(0) \\ e_{\partial, \mathcal{L} x}(0)\end{array}\right]$ the following balance equation is satisfied:

$$
\begin{aligned}
& \frac{1}{2} \frac{d}{d t}\|x(t)\|_{\mathcal{L}}^{2}=\frac{1}{2}\left[\begin{array}{ll}
u^{T}(t) & y^{T}(t)
\end{array}\right] P_{W, \tilde{W}}\left[\begin{array}{l}
u(t) \\
y(t)
\end{array}\right] \\
& -\left\langle G_{0} \mathcal{L} x(t), \mathcal{L} x(t)\right\rangle \\
& \leq \frac{1}{2}\left[u^{T}(t) \quad y^{T}(t)\right] P_{W, \tilde{W}}\left[\begin{array}{l}
u(t) \\
y(t)
\end{array}\right] .
\end{aligned}
$$

Note that the input and output of the system are acting on the boundary of the spatial domain. Also, (12) can be seen as an energy balance equation since $(1 / 2)\|x(t)\|_{\mathcal{L}}^{2}$ usually equals the energy function of the system. Furthermore, the supply rate of energy (the flow of energy through the boundary of the spatial domain) is determined by $P_{W, \tilde{W}}$ and hence by $W$ and $\widetilde{W}$. In fact, this energy balance will help us to prove results for exponential stability for this class of systems. For more information on this type of systems see [14], [16] and [17].

By differentiating the energy along trajectories, or by using (12) and the notation introduced in Theorem II.3 together with (3)-(5), we find that

$$
\begin{aligned}
\frac{1}{2} \frac{d}{d t}\|x(t)\|_{\mathcal{L}}^{2}=\frac{1}{2}\left[\left\langle(\mathcal{L} x)(t, b), P_{1}(\mathcal{L} x)(t, b)\right\rangle_{\mathbb{R}}\right. \\
\left.\quad-\left\langle(\mathcal{L} x)(t, a), P_{1}(\mathcal{L} x)(t, a)\right\rangle_{\mathbb{R}}\right]-\left\langle G_{0} \mathcal{L} x(t), \mathcal{L} x(t)\right\rangle .
\end{aligned}
$$

\section{EXPONENTIAL STABILITY}

In this section we consider the class of BCS introduced in Theorem II.3. Since we are interested in stability, we assume throughout this section that the input function $u(t)=0$ for all $t \geq 0$ and that the energy function of the system, $E$, equals $(1 / 2)\|x(t)\|_{\mathcal{L}}^{2}$. Following this, (12) can be rewritten as

$$
\frac{d}{d t} E(t)=\frac{1}{2}\left\langle P_{W_{2}} y(t), y(t)\right\rangle_{\mathbb{R}}-\left\langle G_{0} \mathcal{L} x(t), \mathcal{L} x(t)\right\rangle
$$

where $P_{W_{2}}$ is the element $(2,2)$ of the block matrix $P_{W, \tilde{W}}$.

To prove the main results of this section we first prove some estimates involving the energy function and the boundary variables. Then, based on those estimates we prove the exponential stability results.

Lemma III.1: Consider a BCS as described in Theorem II.3. If $u(t)=0$, for all $t \geq 0$, then the energy of the system $E(t)=(1 / 2)\|x(t)\|_{\mathcal{L}}^{2}$ satisfies for $\tau$ large enough

$$
\begin{aligned}
E(\tau) & \leq c(\tau) \int_{0}^{\tau}\|(\mathcal{L} x)(t, b)\|_{\mathbb{R}}^{2} d t, \\
\text { and } \quad E(\tau) & \leq c(\tau) \int_{0}^{\tau}\|(\mathcal{L} x)(t, a)\|_{\mathbb{R}}^{2} d t
\end{aligned}
$$

where $c$ is a positive constant that only depends on $\tau$.

Proof: For simplicity let $P_{G}=P_{0}-G_{0}$. Recall that the energy of the system is given by

$$
E(t)=\frac{1}{2} \int_{a}^{b} x^{T}(t, z) \mathcal{L}(z) x(t, z) d z .
$$

To prove the estimates (15) we employ the idea used by Cox and Zuazua in [15]. We define the (positive) function

$$
F(z)=\int_{\gamma(b-z)}^{\tau-\gamma(b-z)} x^{T}(t, z) \mathcal{L}(z) x(t, z) d t
$$


where $\gamma>0, \tau>2 \gamma(b-a)$, and $z \in[a, b]$. It thus follows that (the prime $^{\prime}$ denotes differentiation with respect to $z$ )

$$
\begin{aligned}
& F^{\prime}(z)=\int_{\gamma(b-z)}^{\tau-\gamma(b-z)} x^{T}(t, z) \frac{\partial}{\partial z}(\mathcal{L}(z) x(t, z)) d t \\
& \quad+\int_{\gamma(b-z)}^{\tau-\gamma(b-z)}\left(\frac{\partial}{\partial z} x(t, z)\right)^{T} \mathcal{L}(z) x(t, z) d t \\
& \quad+\gamma x^{T}(\gamma(b-z), z) \mathcal{L}(z) x(\gamma(b-z), z) \\
& \quad+\gamma x^{T}(\tau-\gamma(b-z), z) \mathcal{L}(z) x(\tau-\gamma(b-z), z) .
\end{aligned}
$$

Since $P_{1}$ is nonsingular and $\partial x / \partial t=P_{1}(\partial / \partial z)(\mathcal{L} x)+P_{G} \mathcal{L} x$ we obtain (for simplicity we omit the dependence on $z$ and $t$ )

$$
\begin{aligned}
F^{\prime}(z)= & \int_{\gamma(b-z)}^{\tau-\gamma(b-z)} x^{T} P_{1}^{-1}\left(\frac{\partial x}{\partial t}-P_{G} \mathcal{L} x\right) d t \\
& +\gamma x^{T}(\tau-\gamma(b-z), z) \mathcal{L}(z) x(\tau-\gamma(b-z), z) \\
& +\int_{\gamma(b-z)}^{\tau-\gamma(b-z)}\left(P_{1}^{-1} \frac{\partial x}{\partial t}-\mathcal{L}^{\prime} x-P_{1}^{-1} P_{G} \mathcal{L} x\right)^{T} x d t \\
& +\gamma x^{T}(\gamma(b-z), z) \mathcal{L}(z) x(\gamma(b-z), z) \\
= & \left.x^{T}(t, z) P_{1}^{-1} x(t, z)\right|_{t=\gamma(b-z)} ^{t=\tau-\gamma(b-z)}-\int_{\gamma(b-z)}^{\tau-\gamma(b-z)} x^{T} \mathcal{L}^{\prime} x d t \\
& -\int_{\gamma(b-z)}^{\tau-\gamma(b-z)} x^{T}\left(\mathcal{L} P_{G}^{T} P_{1}^{-1}+P_{1}^{-1} P_{G} \mathcal{L}\right) x d t \\
& +\gamma x^{T}(\tau-\gamma(b-z), z) \mathcal{L}(z) x(\tau-\gamma(b-z), z) \\
& +\gamma x^{T}(\gamma(b-z), z) \mathcal{L}(z) x(\gamma(b-z), z)
\end{aligned}
$$

where we used $P_{1}^{T}=P_{1}, \mathcal{L}^{T}=\mathcal{L}$. By simplifying the equation above one obtains

$$
\begin{aligned}
F^{\prime}(z)= & -\int_{\gamma(b-z)}^{\tau-\gamma(b-z)} x^{T}\left(\mathcal{L} P_{G}^{T} P_{1}^{-1}+P_{1}^{-1} P_{G} \mathcal{L}+\mathcal{L}^{\prime}\right) x d t \\
& +x^{T}(\tau-\gamma(b-z), z)\left[P_{1}^{-1}+\gamma \mathcal{L}(z)\right] x(\tau-\gamma(b-z), z) \\
& +x^{T}(\gamma(b-z), z)\left[-P_{1}^{-1}+\gamma \mathcal{L}(z)\right] x(\gamma(b-z), z) .
\end{aligned}
$$

By choosing $\gamma$ large enough, i.e., by choosing $\tau$ large, we get that $P_{1}^{-1}+\gamma \mathcal{L}$ and $-P_{1}^{-1}+\gamma \mathcal{L}$ are coercive (positive). This in turn implies that (for $\tau$ large enough)

$$
F^{\prime}(z) \geq-\int_{\gamma(b-z)}^{\tau-\gamma(b-z)} x^{T}\left(\mathcal{L} P_{G}^{T} P_{1}^{-1}+P_{1}^{-1} P_{G} \mathcal{L}+\mathcal{L}^{\prime}\right) x d t .
$$

Since $P_{1}, P_{G}$ are constant matrices and, by assumption, $\mathcal{L}^{\prime}(z)$ is bounded, i.e., $x^{T} \mathcal{L}^{\prime}(z) x \leq c x^{T} \mathcal{L}(z) x$, we get using (17)

$$
F^{\prime}(z) \geq-\kappa \int_{\gamma(b-z)}^{\tau-\gamma(b-z)} x^{T}(t, z) \mathcal{L}(z) x(t, z) d t=-\kappa F(z)
$$

where $\kappa$ is a positive constant. Thus we have $F^{\prime}(z) / F(z) \geq-\kappa$, which in turn implies

$$
\begin{aligned}
\int_{z_{1}}^{z_{2}} \frac{F^{\prime}(z)}{F(z)} d z \geq-\kappa \int_{z_{1}}^{z_{2}} d z, \quad \text { for } z_{2} & \geq z_{1} \\
\Rightarrow & \ln \left(F\left(z_{2}\right)\right)-\ln \left(F\left(z_{1}\right)\right) \geq-\kappa\left(z_{2}-z_{1}\right) \\
\Rightarrow F\left(z_{2}\right) & \geq F\left(z_{1}\right) \mathrm{e}^{-\kappa\left(z_{2}-z_{1}\right)} \Rightarrow F(b) \geq F(z) \mathrm{e}^{-\kappa(b-a)}
\end{aligned}
$$

for $z \in[a, b]$. On the other hand, since we have that $E\left(t_{2}\right) \leq E\left(t_{1}\right)$ for any $t_{2} \geq t_{1}$ (by the contraction property of the semigroup), we deduce that

$$
\begin{aligned}
\int_{\gamma(b-a)}^{\tau-\gamma(b-a)} E(t) d t & \geq E(\tau-\gamma(b-a)) \int_{\gamma(b-a)}^{\tau-\gamma(b-a)} d t \\
& =(\tau-2 \gamma(b-a)) E(\tau-\gamma(b-a)) .
\end{aligned}
$$

Using the definition of $F(z)$ and $E(t)$, see (17) and (16), together with the equation above as well as the estimate (18), and the coercivity of $\mathcal{L}$ we obtain

$$
\begin{aligned}
& 2(\tau-2 \gamma(b-a)) E(\tau) \\
& \quad \leq 2(\tau-2 \gamma(b-a)) E(\tau-\gamma(b-a)) \\
& \quad \leq \int_{a}^{b} \int_{\gamma(b-a)}^{\tau-\gamma(b-a)} x^{T}(t, z) \mathcal{L} x(t, z) d t d z \leq \int_{a}^{b} F(z) d z \\
& \quad \leq(b-a) F(b) \mathrm{e}^{\kappa(b-a)} \leq c_{1} \int_{0}^{\tau}\|(\mathcal{L} x)(t, b)\|_{\mathbb{R}}^{2} d t
\end{aligned}
$$

where $c_{1}=(b-a)\left\|\mathcal{L}^{-1}(b)\right\| \mathrm{e}^{\kappa(b-a)}$. Hence for $\tau \gg 0$

$$
E(\tau) \leq c_{2}(\tau) \int_{0}^{\tau}\|(\mathcal{L} x)(t, b)\|_{\mathbb{R}}^{2} d t
$$

where $c_{2}=c_{1} / 2(\tau-2 \gamma(b-a))$. This proves the first estimate on (15). The second estimate follows by replacing $F(z)$ in the argument above by

$$
\tilde{F}(z)=\int_{\gamma(z-a)}^{\tau-\gamma(z-a)} x^{T}(t, z) \mathcal{L}(z) x(t, z) d t .
$$

Theorem III.2: Consider a BCS as described in Theorem II.3 with $u(t)=0$, for all $t \geq 0$. If the energy of the system $E(t)=(1 / 2)\|x(t)\|_{\mathcal{L}}^{2}$ satisfies

$$
\text { either } \begin{aligned}
\frac{d E}{d t}(t) & \leq-k_{1}\|(\mathcal{L} x)(t, b)\|_{\mathbb{R}}^{2} \\
\text { or } \quad \frac{d E}{d t}(t) & \leq-k_{1}\|(\mathcal{L} x)(t, a)\|_{\mathbb{R}}^{2}
\end{aligned}
$$

where $k_{1}$ is a positive real constant, then the system is exponentially stable.

In particular, if the matrix $\alpha=-(1 / 2) P_{W_{2}}$ (see (14)) is positive definite $(\alpha>0)$, then the system is exponentially stable. In other words, if the $(2,2)$-block of the matrix $P_{W, \tilde{W}}$ in (12) is negative definite, then the system is exponentially stable.

Remark III.3: Note that Theorem II.3 provides an explicit expression for $(d E / d t)(t)$, see (12), (13), or (14).

Proof of Theorem III.2: Without loss of generality we assume that the first inequality of (20) holds. Let $\tau$ be the same as in Lemma III.1. From (20) we have that

$$
E(\tau)-E(0) \leq-k_{1} \int_{0}^{\tau}\|(\mathcal{L} x)(t, b)\|_{\mathbb{R}}^{2} d t .
$$

Combining this with (15), we find that

$$
E(\tau)-E(0) \leq \frac{-k_{1}}{c(\tau)} E(\tau)
$$

Thus $E(\tau) \leq c_{0} E(0)$ for some $c_{0}<1$. From this we see that the semigroup $T(t)$ generated by $\mathcal{A}$ (see Theorem II.3) satisfies $\|T(\tau)\|<$ 1 , from which we obtain exponential stability. 
Next we prove the second part of the theorem. To do so, we find a relation between $\|\mathcal{L} x(b)\|_{\mathbb{R}}\left(\right.$ or $\left.\|\mathcal{L} x(a)\|_{\mathbb{R}}\right)$ and $\|y(t)\|_{\mathbb{R}}$. Observe from Theorem II. 3 that (since $u(t)=0$ )

$$
\left[\begin{array}{l}
0 \\
y
\end{array}\right]=\underbrace{\frac{1}{\sqrt{2}}\left[\begin{array}{l}
W \\
W
\end{array}\right]\left[\begin{array}{cc}
P_{1} & -P_{1} \\
I & I
\end{array}\right]}_{M}\left[\begin{array}{l}
(\mathcal{L} x)(b) \\
(\mathcal{L} x)(a)
\end{array}\right] .
$$

Since $P_{1}$ and $\left[\begin{array}{c}W \\ \widetilde{W}\end{array}\right]$ are nonsingular (see Theorem II.3) it follows that the matrix $M$ is invertible and, in particular, $\|M w\|_{\mathbb{R}}^{2} \geq \varepsilon\|w\|_{\mathbb{R}}^{2}$ for some real $\varepsilon>0$. Taking norms on both sides yields

$$
\begin{aligned}
& \|y\|_{\mathbb{R}}^{2}=\left\|M\left[\begin{array}{l}
(\mathcal{L} x)(b) \\
(\mathcal{L} x)(a)
\end{array}\right]\right\|_{\mathbb{R}}^{2} \geq \varepsilon\left\|\left[\begin{array}{l}
(\mathcal{L} x)(b) \\
(\mathcal{L} x)(a)
\end{array}\right]\right\|_{\mathbb{R}}^{2} \geq \varepsilon\|(\mathcal{L} x)(b)\|_{\mathbb{R}}^{2} \\
& \Rightarrow\|(\mathcal{L} x)(b)\|_{\mathbb{R}}^{2} \leq \varepsilon^{-1}\|y\|_{\mathbb{R}}^{2} \quad\left(\text { or }\|(\mathcal{L} x)(a)\|_{\mathbb{R}}^{2} \leq \varepsilon^{-1}\|y\|_{\mathbb{R}}^{2}\right) .
\end{aligned}
$$

From (14) together with the coercivity of $\alpha$, the nonnegativity of $\left\langle G_{0} \mathcal{L} x(t), \mathcal{L} x(t)\right\rangle_{L_{2}}$, and the inequality above yields

$$
\begin{aligned}
\frac{d E}{d t}(t) & =-\langle\alpha y(t), y(t)\rangle_{\mathbb{R}}-\left\langle G_{0} \mathcal{L} x(t), \mathcal{L} x(t)\right\rangle_{L_{2}} \leq-\tilde{k}_{1}\|y(t)\|_{\mathbb{R}}^{2} \\
& \leq-k_{1}\|(\mathcal{L} x)(t, b)\|_{\mathbb{R}}^{2} \quad\left(\text { or }\|(\mathcal{L} x)(t, a)\|_{\mathbb{R}}^{2}\right) .
\end{aligned}
$$

This is the same estimate (20) and hence the system is exponentially stable.

The condition $\alpha=-(1 / 2) P_{W_{2}}>0$ is usually satisfied in systems where damping is applied to the whole boundary. Thus using fullboundary damping almost guarantee an exponentially stable system. However, in practice full-boundary damping is not used often and $\alpha$ is usually a positive semidefinite matrix. In those cases the estimate (20) provides a simple way to prove the exponential stability property.

\section{EXAMPLES}

In this section we show how to apply the results of the previous section. We show that once the input (boundary conditions) and the output are selected, a simple matrix condition allows to conclude on the exponential stability. Also, by using Theorem II. 3 it is easy to select the input and output of the system.

Example 1 (Timoshenko Beam): Consider a flexible beam modeled by the Timoshenko beam equations, see [18], [19] for details on the model and its stabilization. This model can be written as a system (1) by selecting the state variables $x_{1}=\partial w / \partial z-\phi$ (shear displacement), $x_{2}=\rho \partial w / \partial t$ (transverse momentum distribution), $x_{3}=\partial \phi / \partial z$ (angular displacement), $x_{4}=I_{\rho} \partial \phi / \partial t$ (angular momentum distribution). Here $w(t, z)$ is the transverse displacement of the beam and $\phi(t, z)$ is the rotation angle of a filament of the beam. The positive coefficients $\rho(z), I_{\rho}(z), E(z), I(z)$, and $K(z)$ are the mass per unit length, the rotary moment of inertia of a cross section, Young's modulus of elasticity, the moment of inertia of a cross section, and the shear modulus respectively. The energy of the system is known to be

$$
\begin{aligned}
E & =\frac{1}{2} \int_{a}^{b}\left(K\left|x_{1}\right|^{2}+\frac{1}{\rho}\left|x_{2}\right|^{2}+E I\left|x_{3}\right|^{2}+\frac{1}{I_{\rho}}\left|x_{4}\right|^{2}\right) d z \\
& =\frac{1}{2} \int_{a}^{b} x^{T}(z)(\mathcal{L} x)(z) d z=\frac{1}{2}\|x\|_{\mathcal{L}}^{2}
\end{aligned}
$$

where $x=\left[x_{1}, x_{2}, x_{3}, x_{4}\right]^{T}$, and the model of the beam can be written as (see [20])

$$
\begin{aligned}
& \frac{\partial}{\partial t}\left[\begin{array}{l}
x_{1} \\
x_{2} \\
x_{3} \\
x_{4}
\end{array}\right]= \underbrace{\left[\begin{array}{llll}
0 & 1 & 0 & 0 \\
1 & 0 & 0 & 0 \\
0 & 0 & 0 & 1 \\
0 & 0 & 1 & 0
\end{array}\right]}_{P_{1}} \frac{\partial}{\partial z}\left[\begin{array}{c}
K x_{1} \\
\frac{1}{\rho} x_{2} \\
E I x_{3} \\
\frac{1}{I_{\rho}} x_{4}
\end{array}\right] \\
&+\underbrace{\left[\begin{array}{cccc}
0 & 0 & 0 & -1 \\
0 & 0 & 0 & 0 \\
0 & 0 & 0 & 0 \\
1 & 0 & 0 & 0
\end{array}\right]}_{P_{0}}\left[\begin{array}{c}
K x_{1} \\
\frac{1}{\rho} x_{2} \\
E I x_{3} \\
\frac{1}{I_{\rho}} x_{4}
\end{array}\right] .
\end{aligned}
$$

From here we can see that $G_{0}=0, n=4, \mathcal{L}=$ $\operatorname{diag}\left\{K, 1 / \rho, E I, 1 / I_{\rho}\right\}>0$, and $P_{1}$ is nonsingular. Next, according to Definition II.2, we find the port-variables which are given by

$$
\left[\begin{array}{c}
f_{\partial} \\
e_{\partial}
\end{array}\right]=\frac{1}{\sqrt{2}}\left[\begin{array}{c}
\left(\rho^{-1} x_{2}\right)(b)-\left(\rho^{-1} x_{2}\right)(a) \\
\left(K x_{1}\right)(b)-\left(K x_{1}\right)(a) \\
\left(I_{\rho}^{-1} x_{4}\right)(b)-\left(I_{\rho}^{-1} x_{4}\right)(a) \\
\left(E I x_{3}\right)(b)-\left(E I x_{3}\right)(a) \\
\left(K x_{1}\right)(b)+\left(K x_{1}\right)(a) \\
\left(\rho^{-1} x_{2}\right)(b)+\left(\rho^{-1} x_{2}\right)(a) \\
\left(E I x_{3}\right)(b)+\left(E I x_{3}\right)(a) \\
\left(I_{\rho}^{-1} x_{4}\right)(b)+\left(I_{\rho}^{-1} x_{4}\right)(a)
\end{array}\right] .
$$

This beam is usually stabilized by applying velocity feedback. This corresponds to the boundary conditions

$$
\begin{aligned}
& \frac{1}{\rho(a)} x_{2}(a, t)=0, K(b) x_{1}(b, t)=-\alpha_{1} \frac{1}{\rho(b)} x_{2}(b, t), t \geq 0, \\
& \frac{1}{I_{\rho}(a)} x_{4}(a, t)=0, E I(b) x_{3}(b, t)=-\alpha_{2} \frac{1}{I_{\rho}(b)} x_{4}(b, t)
\end{aligned}
$$

where $\alpha_{1}, \alpha_{2} \in \mathbb{R}$ are given positive gain feedback constants. These conditions correspond to a beam clamped at the left side, i.e., at $z=a$, and controlled at $z=b$ by velocity feedback. One may see these boundary conditions as the input of the system (being set to zero). This input can be obtained from the port variables by using the linear combination $W$ given by

$$
W=\frac{1}{\sqrt{2}}\left[\begin{array}{rrrrrrrr}
-1 & 0 & 0 & 0 & 0 & 1 & 0 & 0 \\
0 & 0 & -1 & 0 & 0 & 0 & 0 & 1 \\
\alpha_{1} & 1 & 0 & 0 & 1 & \alpha_{1} & 0 & 0 \\
0 & 0 & \alpha_{2} & 1 & 0 & 0 & 1 & \alpha_{2}
\end{array}\right]
$$

which satisfies

$$
W \Sigma W^{T}=2 \underbrace{\left[\begin{array}{rrrr}
0 & 0 & 0 & 0 \\
0 & 0 & 0 & 0 \\
0 & 0 & \alpha_{1} & 0 \\
0 & 0 & 0 & \alpha_{2}
\end{array}\right]}_{\alpha} .
$$

Example 3.27 of [16] shows how to select an output and find the corresponding $(d / d t) E(t)$ by using Theorem II.3. Here we use (13) to obtain

$$
\frac{d}{d t} E(t)=\left(\frac{K}{\rho} x_{1} x_{2}\right)(b, t)+\left(\frac{E I}{I_{\rho}} x_{3} x_{4}\right)(b, t)
$$




$$
\begin{aligned}
& -\left(\frac{K}{\rho} x_{1} x_{2}\right)(a, t)-\left(\frac{E I}{I_{\rho}} x_{3} x_{4}\right)(a, t) \\
= & -\alpha_{1}\left|\left(\rho^{-1} x_{2}\right)(b, t)\right|^{2}-\alpha_{2}\left|\left(I_{\rho}^{-1} x_{4}\right)(b, t)\right|^{2}
\end{aligned}
$$

where, in the last step, we used the boundary conditions (24). Thus, to check exponential stability is not necessary to define an output. Next, we prove that this system is exponentially stable, and we do this by using Theorem III.2. Using the boundary conditions (24) we obtain

$$
\begin{aligned}
& \|(\mathcal{L} x)(b)\|_{\mathbb{R}}^{2} \\
& =\left|\left(k x_{1}\right)(b)\right|^{2}+\left|\left(\rho^{-1} x_{2}\right)(b)\right|^{2}+\left|\left(E I x_{3}\right)(b)\right|^{2}+\left|\left(I_{\rho}^{-1} x_{4}\right)(b)\right|^{2} \\
& =\left(\alpha_{1}^{2}+1\right)\left|\left(\rho^{-1} x_{2}\right)(b)\right|^{2}+\left(\alpha_{2}^{2}+1\right)\left|\left(I_{\rho}^{-1} x_{4}\right)(b)\right|^{2} \\
& \leq-\kappa \frac{d}{d t} E(t) \quad \text { for some } \kappa>0
\end{aligned}
$$

where we used (27). It is now easy to see that the inequality above is the same estimate (20). Thus, provided that $k(z), \rho(z), E I(z)$, and $I_{\rho}(z)$ are continuously differentiable (see Assumption II.1), we can conclude from Theorem III.2 that the system is exponentially stable.

Example 2 (Heat Exchanger): We consider the following model of counterflow heat exchanger model

$$
\frac{\partial}{\partial t}\left[\begin{array}{l}
\theta_{1} \\
\theta_{2}
\end{array}\right]=\left[\begin{array}{cc}
-v_{1} & 0 \\
0 & v_{2}
\end{array}\right] \frac{\partial}{\partial z}\left[\begin{array}{l}
\theta_{1} \\
\theta_{2}
\end{array}\right]-\underbrace{\left[\begin{array}{cc}
h_{1} & -h_{1} \\
-h_{2} & h_{2}
\end{array}\right]}_{P}\left[\begin{array}{l}
\theta_{1} \\
\theta_{2}
\end{array}\right]
$$

where $\theta_{1}$ and $\theta_{2}$ represent the temperatures at space position $z \in(a, b)$ and time $t>0$, respectively. The positive constants $v_{1}, v_{2}, h_{1}$, and $h_{2}$ are physical parameters, see [21].

Note that this model does not fit into our formalism directly. However, our approach can still be applied by using a linear transformation. To do so, we select the state variable as

$$
x=\left[\begin{array}{l}
x_{1} \\
x_{2}
\end{array}\right]=T^{-1}\left[\begin{array}{l}
\theta_{1} \\
\theta_{2}
\end{array}\right] \text { with } T=\left[\begin{array}{cc}
\sqrt{h_{1}} & 0 \\
0 & \sqrt{h_{2}}
\end{array}\right] .
$$

Thus, it is now easy to see that (28) can be transformed into the similar system

$$
\frac{\partial}{\partial t}\left[\begin{array}{l}
x_{1} \\
x_{2}
\end{array}\right]=\underbrace{\left[\begin{array}{rr}
-v_{1} & 0 \\
0 & v_{2}
\end{array}\right]}_{P_{1}} \frac{\partial}{\partial z}\left[\begin{array}{l}
x_{1} \\
x_{2}
\end{array}\right]-\underbrace{\left[\begin{array}{cc}
h_{1} & -\sqrt{h_{1} h_{2}} \\
-\sqrt{h_{1} h_{2}} & h_{2}
\end{array}\right]}_{G_{0}=T-1 P T}\left[\begin{array}{l}
x_{1} \\
x_{2}
\end{array}\right]
$$

where we now have an operator that fits into our class of systems, see (1), since $G_{0} \geq 0$. In this case we have $\mathcal{L}=I, n=2$, and the boundary port-variables are

$$
\left[\begin{array}{c}
f_{\partial, x} \\
e_{\partial, x}
\end{array}\right]=\frac{1}{\sqrt{2}}\left(\begin{array}{c}
-v_{1} x_{1}(b)+v_{1} x_{1}(a) \\
v_{2} x_{2}(b)-v_{2} x_{2}(a) \\
x_{1}(b)+x_{1}(a) \\
x_{2}(b)+x_{2}(a)
\end{array}\right)
$$

In [21] the authors study the exponential stability of the system (28) under the boundary feedback

$$
\theta_{1}(a)+k_{1} \theta_{2}(a)=0, \quad k_{2} \theta_{1}(b)+\theta_{2}(b)=0
$$

where $k_{1}$ and $k_{2}$ are feedback gains. Here we prove the same result by using Theorem III.2. The boundary conditions above corresponds to the boundary conditions (see (29))

$$
\sqrt{h_{1}} x_{1}(a)+k_{1} \sqrt{h_{2}} x_{2}(a)=0, k_{2} \sqrt{h_{1}} x_{1}(b)+\sqrt{h_{2}} x_{2}(b)=0
$$

for the system (30). To obtain these boundary conditions we can select the matrix $W$ as follows

$$
W=\frac{1}{\sqrt{2}}\left(\begin{array}{cccc}
\frac{\sqrt{h_{1}}}{v_{1}} & -\frac{\sqrt{h_{2}}}{v_{2}} k_{1} & \sqrt{h_{1}} & k_{1} \sqrt{h_{2}} \\
-\frac{\sqrt{h_{1}}}{v_{1}} k_{2} & \frac{\sqrt{h_{2}}}{v_{2}} & k_{2} \sqrt{h_{1}} & \sqrt{h_{2}}
\end{array}\right) .
$$

Thus, for the operator on the right hand side of (30) to generate a contraction semigroup we need $W \Sigma W^{T} \geq 0$, see Theorem II.3. In this case we have,

$$
W \Sigma W^{T}=\left[\begin{array}{cc}
\frac{h_{1}}{v_{1}}-k_{1}^{2} \frac{h_{2}}{v_{2}} & 0 \\
0 & \frac{h_{2}}{v_{2}}-k_{2}^{2} \frac{h_{1}}{v_{1}}
\end{array}\right]
$$

and hence we need

$$
k_{1}^{2} \leq \frac{h_{1} v_{2}}{v_{1} h_{2}} \quad \text { and } \quad k_{2}^{2} \leq \frac{h_{2} v_{1}}{v_{2} h_{1}} .
$$

These are exactly the same conditions imposed in [21] in the analysis of the system (28) with boundary conditions (32). Therefore, if the conditions (35) are satisfied, we have that

$\mathcal{A}_{T}\left[\begin{array}{l}x_{1} \\ x_{2}\end{array}\right]=\left[\begin{array}{rc}-v_{1} & 0 \\ 0 & v_{2}\end{array}\right] \frac{\partial}{\partial z}\left[\begin{array}{l}x_{1} \\ x_{2}\end{array}\right]-\left[\begin{array}{cc}h_{1} & -\sqrt{h_{1} h_{2}} \\ -\sqrt{h_{1} h_{2}} & h_{2}\end{array}\right]\left[\begin{array}{l}x_{1} \\ x_{2}\end{array}\right]$

with domain

$$
D\left(\mathcal{A}_{T}\right)=\left\{x \in H^{1}(a, b)^{2} \mid\left[\begin{array}{l}
f_{\partial}, x \\
e_{\partial}, x
\end{array}\right] \in \operatorname{ker} W\right\}
$$

generates a contraction semigroup. This in turn implies that the corresponding semigroup generator of the system (28) generates a uniformly bounded semigroup, see [9, Exercise 2.5]. Similarly, if the semigroup generated by $\mathcal{A}_{T}$ is exponentially stable, then the system (28) will also be exponentially stable.

Coming back to the system (30), we could select an output. However, as is clear form Theorem III. 2 the stability will not depend on the choice of the output, but on the choice of the input. From (13) and (30), we have that

$$
\begin{aligned}
\frac{d E}{d t}(t)= & \frac{1}{2}\left[-\nu_{1} x_{1}(t, b)^{2}+\nu_{2} x_{2}(t, b)^{2}\right. \\
& \left.+\nu_{1} x_{1}(t, a)^{2}-\nu_{2} x_{2}(t, a)^{2}\right]-\left\langle G_{0} x, x\right\rangle \\
= & \frac{1}{2}\left[\left(-\nu_{1}+\frac{\nu_{2} k_{2}^{2} h_{1}}{h_{2}}\right) x_{1}(t, b)^{2}\right. \\
& \left.+\left(\frac{\nu_{1} k_{1}^{2} h_{2}}{h_{1}}-\nu_{2}\right) x_{2}(t, a)^{2}\right]-\left\langle G_{0} x, x\right\rangle
\end{aligned}
$$

where we used condition (33). Note from (33) that $\|(\mathcal{L} x)(b)\|_{\mathbb{R}}^{2}=$ $\left(1+k_{2}^{2} h_{1} / h_{2}\right)\left|x_{1}(b)\right|^{2}$ and $\|(\mathcal{L} x)(a)\|_{\mathbb{R}}^{2}=\left(1+k_{1}^{2} h_{2} / h_{1}\right)\left|x_{2}(a)\right|^{2}$. Hence by using Theorem III.2 we see that if $k_{1}^{2}<h_{1} v_{2} / v_{1} h_{2}$ and $k_{2}^{2} \leq h_{2} v_{1} / v_{2} h_{1}$ or $k_{1}^{2} \leq h_{1} v_{2} / v_{1} h_{2}$ and $k_{2}^{2}<h_{2} v_{1} / v_{2} h_{1}$, then the system (30) with boundary conditions (33) is exponentially stable. Since (35) is assumed to hold, we can conclude that if

$$
\text { either } k_{1}^{2}<\frac{h_{1} v_{2}}{v_{1} h_{2}} \text {, or } k_{2}^{2}<\frac{h_{2} v_{1}}{v_{2} h_{1}} \text {, }
$$

the system is exponentially stable. Note that the conclusion above includes the cases $k_{1}=0$ and/or $k_{2}=0$. Let us briefly compare the above conclusion with the results in [21]. In [21], the authors prove that the system is exponentially stable if either $k_{1}^{2}=h_{1} v_{2} / v_{1} h_{2}$ and $k_{2}^{2}=h_{2} v_{1} / v_{2} h_{1}$ or $k_{1}^{2}=h_{1} v_{2} / v_{1} h_{2}$ and $k_{2}=0$. They do this by finding, for each case, some estimates on the eigenvalues of $\mathcal{A}_{T}$ and 
its resolvent operator. For the remaining cases satisfying (35) the authors suggest in the conclusion solving numerically an equation that is related to the eigenvalues of the system. Although Theorem III.2 excludes the case $k_{1}^{2}=h_{1} v_{2} / v_{1} h_{2}$ and $k_{2}^{2}=h_{2} v_{1} / v_{2} h_{1}$, it has the advantage of treating all the remaining cases satisfying (35) using only a matrix inequality.

\section{CONCLUSION}

We provided tools that facilitate checking the exponentially stability property of a class of BCS. We showed that by using results of [14] and [16] it is easy to select the input and outputs of a BCS. Therefore, we use those results on boundary port Hamiltonian systems to define inputs and outputs for our class of BCS. Once this is done, checking for exponential stability follows easily. The main idea behind the proof consists in using a multiplier common to the whole class of BCS. This multiplier only depends on the norm of the co-energy variables at the boundary of the spatial domain. In this way one avoids searching for different multipliers every time the system or the boundary conditions are changed. This simplifies drastically the verification of the exponential stability property, as can be seen already from the examples in Section IV. Also the proof of the results of [22] and [23] can be simplified by using our results.

Even though the results are only valid for a class of one-dimensional systems, the authors believe that the approach has potential to be extended to 2-D and 3-D systems. The key point being the definition and selection of the boundary port variables. Some ideas about this are presented in [16, Ch. 8]. However, this still requires more research.

\section{REFERENCES}

[1] H. O. Fattorini, "Boundary control systems," SIAM J. Control, vol. 6, pp. 349-385, 1968.

[2] A. V. Balakrishnan, "Applications of Mathematics," in Applied Functional Analysis. New York: Springer-Verlag, 1976, vol. 3.

[3] A. V. Balakrishnan, "Boundary control of parabolic equations: L-Q-R theory," in Theory of Nonlinear Operators. Berlin, Germany: Akademie-Verlag, 1978, vol. 6, pp. 11-23.

[4] J.-L. Lions, Optimal Control of Systems Governed by Partial Differential Equations Transl.:from the French by S. K. Mitter. Die Grundlehren der mathematischen Wissenschaften. New York: Springer-Verlag, 1971.

[5] I. Lasiecka and R. Triggiani, Control Theory for Partial Differential Equations: I-II. Cambridge, U.K.: Cambridge University Press, 2000, vol. 74-75.

[6] R. F. Curtain and A. J. Pritchard, "An abstract theory for unbounded control action for distributed parameter systems," SIAM J. Control Optim., vol. 15, no. 4, pp. 566-611, 1977.

[7] R. F. Curtain and A. J. Pritchard, Infinite Dimensional Linear Systems Theory. Berlin, Germany: Springer-Verlag, 1978, vol. 8.

[8] A. J. van der Schaft and B. M. Maschke, "Hamiltonian formulation of distributed-parameter systems with boundary energy flow," J. Geometry Phys., vol. 42, pp. 166-194, 2002.

[9] R. F. Curtain and H. J. Zwart, An Introduction to Infinite-Dimensional Linear Systems Theory. New York: Springer-Verlag, 1995.

[10] A. Bensoussan, G. Da Prato, M. C. Delfour, and S. K. Mitter, Representation and Control of Infinite Dimensional Systems, second ed. Boston, MA: Birkhäuser, 2007.

[11] V. Komornik and P. Loreti, Fourier Series in Control Theory. New York: Springer-Verlag, 2005.

[12] V. Komornik, Exact Controllability and Stabilization. The Multiplier Method. Paris, France: Masson, 1994

[13] Z. H. Luo, B. Z. Guo, and O. Morgul, Stability and Stabilization of Infinite Dimensional Systems with Applications. New York: SpringerVerlag, 1999.

[14] Y. Le Gorrec, H. Zwart, and B. Maschke, "Dirac structures and boundary control systems associated with skew-symmetric differential operators," SIAM J. Control Optim., vol. 44, no. 2, pp. 1864-1892, 2005.

[15] S. Cox and E. Zuazua, "The rate at which energy decays in a string damped at one end," Indiana Univ. Math. J., vol. 44, no. 2, pp. 545-573, 1995.
[16] J. A. Villegas, "A Port-Hamiltonian Approach to Distributed Parameter Systems," Ph.D. dissertation, Department of Applied Mathematics, University of Twente,, Enschede, The Netherlands, 2007.

[17] J. A. Villegas, H. Zwart, and A. J. van der Schaft, "Port representations of the transmission line," in Proc. IFAC World Congress, Jul. 2005, [CD $\mathrm{ROM}]$.

[18] G. Q. Xu, "Boundary feedback exponential stabilization of a Timoshenko beam with both ends free," Int. J. Control, vol. 78, no. 4, pp. 286-297, 2005.

[19] J. U. Kim and Y. Renardy, "Boundary control of the Timoshenko beam," SIAM J. Control Optim., vol. 25, no. 6, pp. 1417-1429, 1987.

[20] G. Golo, V. Talasila, and A. J. van der Schaft, "A Hamiltonian formulation of the Timoshenko beam model," in Proc. Mechatronics'02, Jun. 2002, [CD ROM].

[21] N. Kunimatsu and H. Sano, "Stability analysis of heat-exchanger equations with boundary feedbacks," IMA J. Math. Control Inform., vol. 15, pp. 317-330, 1998.

[22] H. Sano and N. Kunimatsu, "On the stability of a linear bioprocess model with recycle loop," IEEE Trans. Automat. Control, vol. 50, no. 8, pp. 1200-1205, Aug. 2005.

[23] C.-G. Zhang, "Boundary feedback stabilization of the undamped Timoshenko beam with both ends free," J. Math. Anal. Appl., vol. 326, no. 1, pp. 488-499, 2007.

\section{Delay-Dependent Exponential Stability of Neutral Stochastic Delay Systems}

\author{
Lirong Huang and Xuerong Mao
}

\begin{abstract}
This technical note studies stability of neutral stochastic delay systems by linear matrix inequality approach. Delay-dependent criterion for exponential stability is presented and numerical examples are conducted to verify the effectiveness of the proposed method.
\end{abstract}

Index Terms-Exponential stability, linear matrix inequalitys (LMIs), neutral systems, stochastic systems, time delay.

\section{INTRODUCTION}

Many dynamical systems are described with neutral functional differential equations that include neutral delay differential equations [19]. These systems are called neutral-type systems or neutral systems. Motivated by chemical engineering systems as well as theory of aero elasticity, studies on deterministic neutral systems have been of research interest over the past decades [3]-[11], [21]. As stochastic modelling has come to play an important role in many branches of science and industry, neutral stochastic delay systems have been intensively studied over recent year [10]-[17]. Mao [14]-[17] initiated the study of exponential stability of neutral stochastic functional equations, developed the Razumikhin-type theorems further for exponential stability of neutral stochastic functional equations and studied asymptotic properties of neutral stochastic delay differential equations [1]. More recently, Luo et al. [12] proposed new criteria on exponential stability of neutral stochastic delay differential equations while Chen et al. [2] studied delay-dependent stability of neutral stochastic delay systems. However, the stability result in [2] employed an

Manuscript received July 22, 2008; revised September 03, 2008 and September 23, 2008. Current version published January 14, 2009. This work was supported by UK ORSAS and the University of Strathclyde. Recommended by Associate Editor J.-F. Zhang.

The authors are with the Department of Statistics and Modelling Science, University of Strathclyde, Glasgow G1 1XH, U.K. (e-mail: lirong@stams.strath.ac.uk; xuerong@stams.strath.ac.uk).

Digital Object Identifier 10.1109/TAC.2008.2007178 